\title{
Inter-rater reliability of Electronic Head Posture Instrument (EHPI) for measuring Cranio-vertebral angle in young adults
}

\author{
V. Karthik ${ }^{1}$, S. Arulpragassame ${ }^{2}$, K. Parkavi ${ }^{3}$, S. Harshini ${ }^{4}$ \\ ${ }^{1}$ Research scholar, Lecturer in Physiotherapy. Email: kar_1822003@yahoo.com \\ ${ }^{2}$ Lecturer in Physiotherapy. Email: arulphysio77@gmail.com \\ ${ }^{3}$ Lecturer in Physiotherapy. Email: parkavikarthik2010@gmail.com. \\ ${ }^{4}$ Physiotherapist. Email: harshinidesan469@gmail.com \\ Department of Physical Medicine \&Rehabilitation, Rajah Muthiah Medical College \& \\ Hospital, Annamalai University, Annamalai Nagar, Chidambaram, Tamilnadu, India- \\ 608002 .
}

\author{
Author for correspondence: \\ V. Karthik, MPT (Orthopaedics) \\ Research Scholar, Department of Physical Medicine \& Rehabilitation, \\ Rajah Muthiah Medical College \& Hospital, Annamalai University. \\ Annamalai Nagar, Chidambaram - 608 002, \\ Tamilnadu - India. \\ Mobile: 91- 9843677795. \\ Email: kar_1822003@yahoo.com
}

\begin{abstract}
Purpose: Forward head posture and degree of anterior translation of head can be assessed using Cranio-vertebral angle (CV angle). Recent literatures use Electronic Head Posture Instrument (EHPI) to measure CV angle without exposing the patient to $X$-ray. Objective and Study design: This observational study was cross-sectionally designed and aimed to find out the reliability of Electronic Head Posture Instrument (EHPI) in measuring Cranio-Vertebral Angle of young adults. Methods: 100 apparently healthy young adults were assessed for the $C V$ angle by two Physiotherapy student investigators who are skilled in measuring CV angle using EHPI in two separate sessions of 20 minutes gap with the same instrument. The measurements of $C V$ angle by the two investigators were documented and taken for statistical analysis. Results: The mean difference in the Cranio-Vertebral Angle between the two measurements was 0.46 (+/-) 0.79. The inter-class correlation coefficient for inter-rater reliability of Cranio-Vertebral Angle (CV Angle) by Electronic Head Posture Instrument (EHPI) was 0.925, with the corresponding $P=0.001$. Conclusion: The study results exhibit a significant strong positive correlation between the two measurements supporting a high reliability of EHPI in measuring CV Angle.
\end{abstract}

KEY WORDS: Electronic Head Posture Instrument, Non-specific neck pain, Forward Head Posture, Anterior Head Translation, Cranio Vertebral Angle. 


\section{Introduction}

The use of smartphones for games and surfing internet rather than communication is obvious. Young generation uses smartphones at very early age. This makes alterations in head on neck posture [1]. Forward head posture (FHP) is the most adopted posture of young adults those who spend more time on smartphones, laptops or personal computers $[2,3]$. Various ailments of head, neck and shoulder like myalgia, migraines, cervicogenic headache are expected out of FHP in young age [4-6].

Severity of symptoms is directly proportional to the time spend on smartphones [7]. The centre of the gravity was shifted as the head moves forward. To compensate for this shift in the centre of the gravity, upper body drifts backward and shoulders slump forward so that the head is placed anterior to the trunk [8,9]. It pre disposes the individuals towards pathological conditions like temporomandibular disorders, vertebral bodies disorders, soft tissues length and strength alteration, or even scapula and shoulder dyskinesis $[6,10]$.

Because of these associated problems, assessment of head posture has become increasingly gaining the importance in clinical practices. It helps in evaluating and designing treatment regimens for patients with neck pain. The biomechanical variation due to forward head posture can be best predicted by measuring the Cranio-vertebral angle [11]. It is the angle formed by a horizontal line drawn by through the spinous process of the seventh cervical (C7) vertebra and a line joining the spinous process of $\mathrm{C} 7$ vertebra with the tragus of the ear $[12,13]$.

Some studies used lateral photographic imaging to measure CV angle [11,12]. Herman et al compared the CV angle obtained by assessing the anterior translation of head in lateral cervical radiographs with the measurement of Electronic Head Posture Instrument (EHPI) in patients with mechanical neck pain and suggested EHPI as a valid tool to assess cervical posture [13]. Exposing the patients to radiation need a hospital setup and inconvenient to use in all places. Measuring CV angle by EHPI scale is accurate to one decimal place and the Electronic sensor reads the angle automatically [14,15]. This study focuses to estimate the Inter-rater reliability of Electronic Head Posture Instrument (EHPI) in measuring $\mathrm{CV}$ angle of young adults. We hypothesize that the measurements of EHPI accurately represents the $\mathrm{CV}$ angle.

\section{Materials and Methods}

This observational cross-sectional study was carried out in the Department of Physical Medicine and Rehabilitation, RMMCH at Annamalai University, Chidambaram. The samples were recruited through non-probability sampling method and their informed written consent was obtained. Inclusion: apparently healthy young adults, aged between 20-30 years, both gender. Exclusion: history of neck and back pain for at least 12 months, Visual deficits, diagnosed balance disorders, musculoskeletal pathologies, displayed functional \& structural scoliosis, Excessive thoracic kyphosis. 
100 subjects were assessed for their CV angle by two physiotherapy students who were skilled in measuring CV angle using EHPI at two separate sessions. The sessions were apart by 20 minutes of the same day. Both the skilled assistants were unaware of other's data. The two measurements were documented separately and taken for further statistical analysis of inter- rater reliability.

\section{Procedure of measuring CV angle using EHPI}

$\mathrm{CV}$ angle was measured by the electronic head posture instrument, an electronic angle finder mounted on a transparent plastic base and a tripod camera stand. The C7 spinous process was palpated and identified and an adhesive pin marker was attached over its midpoint of the most prominent part. The participant was them asked with his/her left shoulder in front of the EHPI.

Another pin marker was fixed at the tragus of the ear. Participants were instructed to stand/sit comfortably with his/her weight distribution evenly on both feet and keep the eyes looking straight forward. A virtual line was drawn between the two pin markers from midpoint of the tragus to C7 [13].

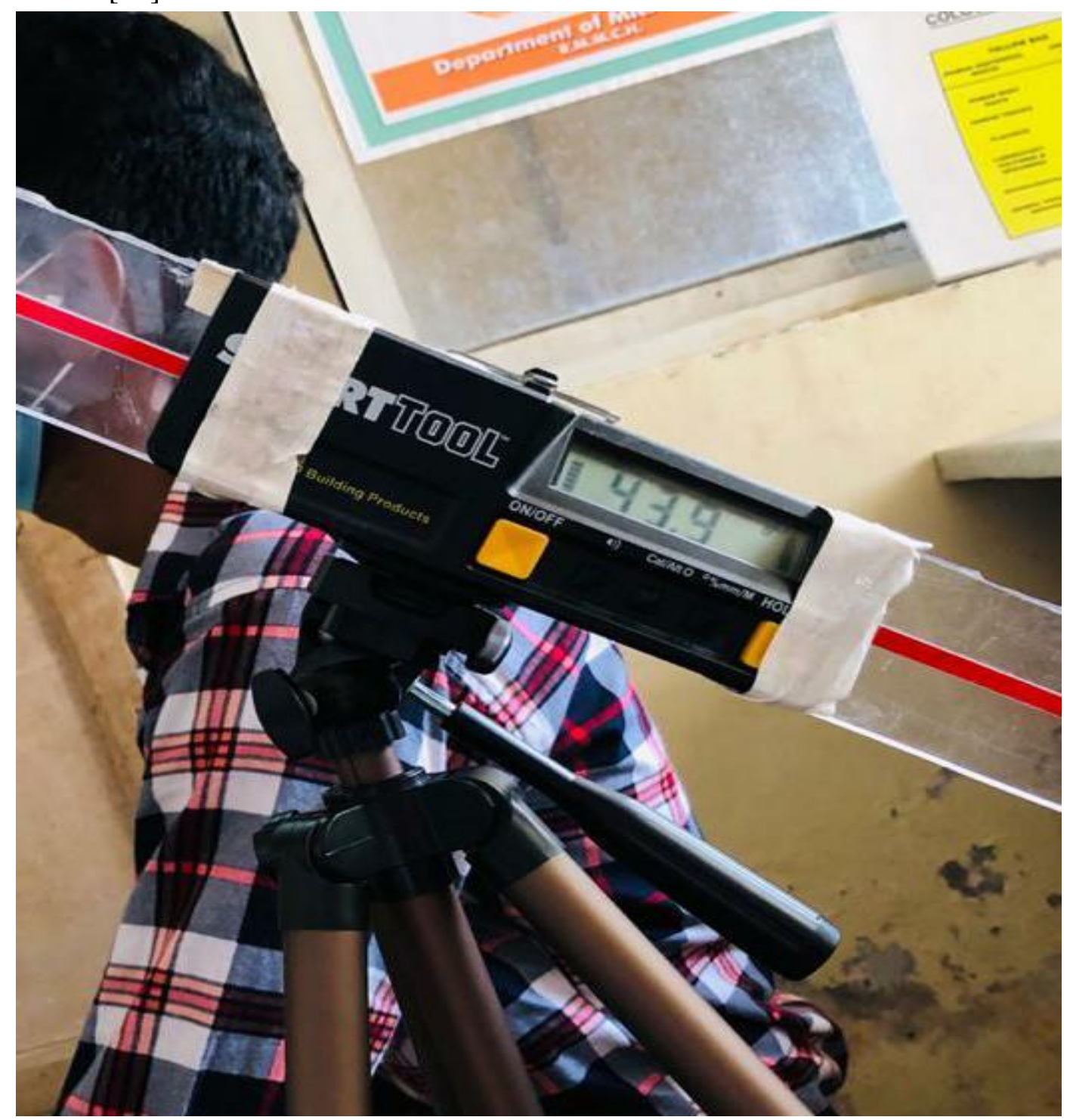




\section{Statistical analysis}

Descriptive statistics in terms of mean, standard deviation of CV angle measured by rater-1 and rater- 2 was made. The mean difference in the $\mathrm{CV}$ angle obtained from two assessments was analyzed using interclass correlation co-efficient method. The statistical analysis was made using Statistical Package for Social Sciences -SPSS Version. 21.

\section{Results:}

To satisfy the purpose a sample of 100 young adults were recruited. Among them 52 were males and 48 were females. Their CV angle were statistically analysed and the following results were drawn.

Table 1: Descriptive statistics of CV angle.

\begin{tabular}{|c|c|c|}
\hline CV angle & Mean & SD \\
\hline Rater 1 & 46.67 & 2.33 \\
\hline Rater 2 & 47.13 & 2.42 \\
\hline Difference in CV Angle & 0.46 & 0.79 \\
\hline
\end{tabular}

(CV angle values were in degrees)

It was observed from table 1 that, the mean value of $\mathrm{CV}$ angle measured by rater one was 46.6 degrees and rater two was 47.13 degrees. The mean difference in the Cranio-Vertebral Angle between the two measurements was $0.46(+/-) 0.79$ degrees.

Table 2: Reliability of EHPI in measuring Cranio-vertebral angle.

\begin{tabular}{|c|c|c|}
\hline Cranio - vertebral angle & $\begin{array}{c}\text { Inter-class correlation } \\
\text { coefficient }\end{array}$ & $\begin{array}{c}p \text {-value } \\
(<0.05)\end{array}$ \\
\hline Head posture instrument & 0.925 & 0.001 \\
\hline
\end{tabular}

$\mathrm{P}<0.005$ is significant.

It is inferred from table 2, that the inter-class correlation coefficient for inter-rater reliability of Cranio-Vertebral Angle (CV Angle) by Electronic Head Posture Instrument (EHPI) was 0.925, with the corresponding $\mathrm{p}=0.001$.

\section{Discussion}

Poor head posture is generally accepted as one of the causes for neck pain because poor postural awareness and habitual poor postural may result in greater loading and the supporting structure and may cause sensitization and pain currently, upright posture evaluation is recommended as part of the comprehensive physical examination of the cervical spine. However, head posture is difficult concept to capture, which is usually assessed subjectively or by complicated procedures. A clinically convenient, reliable and valid assessment tool for head posture needs to be developed. 
The present study evaluated the inter-rater reliability of measuring cranio-vertebral angle using electronic head posture instrument (EHPI) by two well trained physiotherapists. A total of 100 samples satisfied the selection criteria were included and they were assessed CV angle using EHPI and recorded. The result of the study showed a high positive correlation between the two measurements. The obtained results coincides with Hermam mun, et.al who found that the good reverse co-relation $(r=0.72)$ between the EHPI measurement of CV angle and radiological measurement of AHT and supported that head posture assessment should be included in the clinical assessment for patients with neck or upper back pain [13]. The study done by Yip et al concluded that CV angle correlated negatively with neck pain questionnaire and NPRS score and recommend the clinicians to pay special attention to patients with smaller CV angle as they were prone to higher pain intensity and neck disability [8].

The present study was limited to young adults and healthy population. Further studies on various neck disorders and comparing the CV angle obtained in EHPI with angle formed in X-ray can be carried out.

\title{
Conclusion
}

There was a significant strong positive correlation of 0.925 was observed between the two measurements of Cranio-vertebral angle assessed using Electronic Head Posture Instrument. The mean difference in the two readings was 0.46 degrees which was negligible. The result signifies the reliability of Electronic Head Posture Instrument to assess the CV angle. Therefore, the study recommends the use of EHPI by Physiotherapists to assess the CV angle without complex instruments at any type of clinical setup.

\section{Ethical Approval}

The study protocol got ethical clearance from the Institutional Human Ethics Committee, Rajah Muthiah Medical College, Annamalai University.

\author{
Source of support - Self. \\ Conflict of interest - None
}

\section{Acknowledgement}

We would like to acknowledge Dr. Dhanpal Singh, Professor \& Head, PMR $\mathrm{RMMCH}$ for his constant support and all our participants those who willingly participated in the study.

\section{References}

1. Priyal P.Shah,Megha S. Sheth. Correlation of smart phone use addiction with text neck syndrome and SMS thumb in physiotherapy students. Int J Community Med Public Health 2018; 5(6).

2. Nesreen Fawzy Mahumoud, Karima A Hassan, Salwa F Abdelmajeed, Ibraheem M. Moustafa, Anabela $G$ Silva. The relationship between forward head posture and neck 
pain: A systematic review and meta-analysis. Current review in musculoskeletal medicine 2019; 12: 562-577.

3. Parisa Nejati, Sara Lotfian, Azar Moezy, Minanejati. The study of correlation between forward head posture and neck pain in iranian office workers. International Journal of Occupational Medicine and Environmental Health-2015;28(2)

4. Apurva Nitin Worlikar,Dr Mayuri Rajesh Shah. Incidence of forward head posture and associated problems in desktop users.International journal of health sciences and research 2019; 9.

5. Dae-Hyunkim,Chang-Ju Kim,Sung-Min Son.Neck pain in adults with forward head posture: Effects of cranio-vertebral angle and cervical range of motion.Osong public health and research perspectives 2018;9:309-313.

6. Edrish Safiee Contractor, Sweety Shah, Stuti Jayesh Shah. To study the correlation between neck pain and cranio-vertebral angle in young adults. International Archives of Integrated Medicine 2018; 5(4): 81-86.

7. Sang In Jung,Nakyung Lee,Do Youn Lee. The effect of smart phone usage time on posture and respiratory function.Journal of physical therapy science 2016;28(1):186-189.

8. Chris Ho Ting yip, Thomas Tai Wingchiu, Anthony Tung Kuenpoon. The relationship between head posture and severity and disability of patients with neck pain. Manual therapy 2008; 13:148-154.

9. Shivani Lalit Kumar Verma,Jilanishaih,Ranveer Kumar Mahato,Megha Sandeep Sheth-A Cross Sectional Study. Applied Medicl Research 2018;(4)18-21.

10. Seokhyun Nam, Sung Min Son,Nakyung Lee. The intra and inter rater reliabilities of the forward head posture assessments of normal healthy subjects. Journal of physical therapy science 2013; 25(6)737-739.

11. Dalia Mohammed Mosaad, Amr Almaz Abdel-Aziem, Ghada Ismail Mohamed, Enas Anwar Abd Elaty, Karimasalah Mohammed. Effect of forward head and rounded shoulder posture on hand grip strength in asymptomatic young adults. Bulletin faculty of Physical Therapy-2020; 25:5.

12. Rupali Salvi, Sneha Battin. Correlation of mobile phone addiction scale (MPAS) score with craniovertebral angle, scapular index and becks depression inventory score in young adults. Int $j$ physiother 2018; 5:7-14.

13. Herman Mun Cheunglau, Thomas Tai Wing Chiu, Tai Hing Lam. Measurement of craniovertebral angle with electronic head posture instrument: Criterion Validity. Journal of rehabilitation research and development 2010; 47.

14. Edrish Safiee Contractor, Sweety Shah, Stutishah, Parita dave. Validity and reliability of electronic head posture instrument for measurement of cranio-vertebral angle, craniohorizontal angle and sagittal shoulder angle. Indian journal of public health research \&development 2020; 11(7): 260-265.

15. Jinal A. Mamania, Deepak B Anap, Dhanashree Tanksale. Validity and reliability of protractor smart phone application for Measurement of cranio-vertebral and craniohorizontal angle. International journal of physiotherapy 2017; 4(4).

16. JYK Goh, S O Leary, A Chow, T Rusell, S Mcphail. The Relationship between Forward Head Posture and Cervical Muscle Performance in Healthy Individuals. WCPT congress 2015; 101. 